\title{
A Picture for Complex Stochastic Boolean Systems: The Intrinsic Order Graph ${ }^{\star}$
}

\author{
Luis González \\ University of Las Palmas de Gran Canaria, \\ Department of Mathematics, Research Institute IUSIANI, \\ 35017 Las Palmas de Gran Canaria, Spain \\ luisglez@dma.ulpgc.es \\ http://www.dma.ulpgc.es/profesores/personal/lgs/
}

\begin{abstract}
Complex stochastic Boolean systems, depending on a large number $n$ of statistically independent random Boolean variables, appear in many different scientific, technical or social areas. Each one of the $2^{n}$ binary states associated to such systems is denoted by its corresponding binary $n$-tuple of $0 \mathrm{~s}$ and $1 \mathrm{~s},\left(u_{1}, \ldots, u_{n}\right)$, and it has a certain occurrence probability $\operatorname{Pr}\left\{\left(u_{1}, \ldots, u_{n}\right)\right\}$. The ordering between the $2^{n}$ binary $n$ tuple probabilities, $\operatorname{Pr}\left\{\left(u_{1}, \ldots, u_{n}\right)\right\}$, can be illustrated by a directed graph which "scales" them by decreasing order, the so-called intrinsic order graph. In this context, this paper provides a simple algorithm for iteratively drawing the intrinsic order graph, for any complex stochastic Boolean system and for any number $n$ of independent random Boolean variables. The presentation is self-contained.
\end{abstract}

\section{Introduction}

This paper deals with the modelling of complex stochastic Boolean systems, that is, those complex systems which depend on a large number $n$ of random Boolean variables $x_{1}, \ldots, x_{n}$. These systems, with very simple components (the Boolean variables $x_{i}$ only take two possible values: 0,1 ) but complex overall behavior, can be found in many different knowledge areas: Wherever a stochastic Boolean phenomenon with $n$ basic variables appears (Biology, Biochemistry, Climatology, Computer Science, Networks, Engineering, Economics, Sociology, etc.).

According to the usual terminology in Statistics, a stochastic Boolean system can be modeled by the $n$-dimensional Bernoulli distribution (see, e.g., 6]) This distribution consists on $n$ random variables $x_{1}, \ldots, x_{n}$, which only take two possible values, 0 or 1 , with probabilities

$$
\operatorname{Pr}\left\{x_{i}=1\right\}=p_{i}, \quad \operatorname{Pr}\left\{x_{i}=0\right\}=1-p_{i} \quad(1 \leq i \leq n),
$$

so that the sample space is the set $\{0,1\}^{n}$ of the $2^{n}$ binary $n$-tuples (binary strings $), u=\left(u_{1}, \ldots, u_{n}\right)$, of 0 s and 1 s. Throughout this work, we assume that

\footnotetext{
* Partially supported by MEC (Spain) and FEDER. Grant contract: CGL2004-06171C03-02/CLI.
} 
the random variables $x_{i}$ are mutually independent, so that the occurrence probability of each binary $n$-tuple can be easily computed as the product

$$
\operatorname{Pr}\left\{\left(u_{1}, \ldots, u_{n}\right)\right\}=\prod_{i=1}^{n} \operatorname{Pr}\left\{x_{i}=u_{i}\right\}=\prod_{i=1}^{n} p_{i}^{u_{i}}\left(1-p_{i}\right)^{1-u_{i}},
$$

that is, $\operatorname{Pr}\left\{\left(u_{1}, \ldots, u_{n}\right)\right\}$ is the product of factors $p_{i}$ if $u_{i}=1,1-p_{i}$ if $u_{i}=0$.

The following natural question immediately arises in the study of stochastic Boolean systems: How can we order the $2^{n}$ binary strings $\left(u_{1}, \ldots, u_{n}\right)$ by decreasing/increasing order of their occurrence probabilities? Obviously, this question has a relevant, theoretical and practical, interest. However, in spite of the simplicity of Equation (1) to compute the probabilities $\operatorname{Pr}\left\{\left(u_{1}, \ldots, u_{n}\right)\right\}$, this is not a simple question due to its exponential nature. To avoid this obstacle, in [3, 4] the authors have established a simple positional criterion, the so-called Intrinsic Order Criterion (IOC), that a priori assures us that for certain pairs of binary $n$-tuples $u, v \in\{0,1\}^{n}$ (exactly for those pairs whose bits satisfy IOC), the inequality $\operatorname{Pr}\{u\} \geq \operatorname{Pr}\{v\}$ intrinsically holds, that is, $\operatorname{Pr}\{u\} \geq \operatorname{Pr}\{v\}$ for any values of the parameters $p_{i}$ satisfying certain non restrictive hypothesis. Moreover, in 1, 2, the author has "roughly" constructed the intrinsic order graph for the first values of $n$, just by direct application of IOC. However, a general method for easily constructing this graph for all $n \in \mathbb{N}$, has not yet been provided.

In this context, the main goal of this paper is to provide a simple algorithm for recursively drawing the intrinsic order graph, for any number $n$ of Boolean variables. This directed graph is a very useful picture for all complex stochastic Boolean systems (related to any knowledge area), because it "scales" the binary $n$-tuples of $0 s$ and $1 s,\left(u_{1}, \ldots, u_{n}\right) \in\{0,1\}^{n}$, by decreasing order of their occurrence probabilities. In Sect. 2, we describe all previous results required for making this paper self-contained. The rest of the paper is devoted to our new approach. In Sect. 3, we present a simple characterization of the covering relation of the intrinsic order. Finally, in Sect. 4, from this characterization we derive the algorithm for drawing the intrinsic order graph.

\section{The Intrinsic Order}

In 3, 4, we have established the following characterization theorem that allows us to compare two given binary string probabilities, $\operatorname{Pr}\{u\}$ and $\operatorname{Pr}\{v\}$, without computing them.

Theorem 1 (The intrinsic order theorem). Let $x_{1}, \ldots, x_{n}$ be $n$ independent Bernoulli variables, with parameters $p_{i}=\operatorname{Pr}\left\{x_{i}=1\right\} \quad(1 \leq i \leq n)$ satisfying:

$$
0<p_{1} \leq \cdots \leq p_{n} \leq \frac{1}{2}
$$

Then, the probability of the $n$-tuple $\left(u_{1}, \ldots, u_{n}\right) \in\{0,1\}^{n}$ is intrinsically greater than or equal to the probability of the $n$-tuple $\left(v_{1}, \ldots, v_{n}\right) \in\{0,1\}^{n}$ (that is, for 
all set of parameters $\left\{p_{i}\right\}_{i=1}^{n}$ such that (2)) if, and only if, the matrix

$$
M_{v}^{u}:=\left(\begin{array}{lll}
u_{1} & \ldots & u_{n} \\
v_{1} & \ldots & v_{n}
\end{array}\right)
$$

either has no $\left(\begin{array}{l}1 \\ 0\end{array}\right)$ columns, or for each $\left(\begin{array}{l}1 \\ 0\end{array}\right)$ column there exists (at least) one corresponding preceding $\left(\begin{array}{l}0 \\ 1\end{array}\right)$ column $($ IOC $)$.

Remark 1. In the following, we assume that the parameters $p_{i}$ always satisfy condition (2). Note that this hypothesis is not restrictive for practical applications because, if for some $i: p_{i}>\frac{1}{2}$, then we only need to consider the variable $\overline{x_{i}}=1-x_{i}$, instead of $x_{i}$. Next, we order the $n$ Bernoulli variables by increasing order of their probabilities.

Remark 2. The $\left(\begin{array}{l}0 \\ 1\end{array}\right)$ column preceding to each $\left(\begin{array}{l}1 \\ 0\end{array}\right)$ column is not required to be necessarily placed at the immediately previous position, but just at previous position.

Remark 3. The term corresponding, used in Theorem 1 has the following meaning: For each two $\left(\begin{array}{l}1 \\ 0\end{array}\right)$ columns in matrix $M_{v}^{u}$, there must exist (at least) two different $\left(\begin{array}{l}0 \\ 1\end{array}\right)$ columns preceding to each other. In other words, IOC requires that for each $\left(\begin{array}{l}1 \\ 0\end{array}\right)$ column $C$ in matrix $M_{v}^{u}$, if we denote by $n_{1}^{0}(C)\left(n_{0}^{1}(C)\right.$, resp.) the number of $\left(\begin{array}{l}0 \\ 1\end{array}\right)$ columns $\left(\left(\begin{array}{l}1 \\ 0\end{array}\right)\right.$ columns, resp.) preceding $C$, then $n_{1}^{0}(C)>n_{0}^{1}(C)$.

Theorem 1 naturally leads to the following partial order relation on the set $\{0,1\}^{n}$. The so-called intrinsic order (because it only depends of the relative positions of $0 \mathrm{~s}$ and $1 \mathrm{~s}$ in the binary $n$-tuples $u, v$, but not on the basic probabilities $\left.p_{i}\right)$, will be denoted by "§”, and we shall denote by $P_{n}$ the partially ordered set (poset, for short) $\left(\{0,1\}^{\bar{n}}, \preceq\right)$. See [5] for more details about posets.

Definition 1. For all $u, v \in\{0,1\}^{n}$

$u \succeq v$ iff $\operatorname{Pr}\{u\} \geq \operatorname{Pr}\{v\}$ for all set $\left\{p_{i}\right\}_{i=1}^{n}$ s.t. (2) iff $M_{v}^{u}$ satisfies IOC.

Example 1. $(0,0,1,1) \succeq(1,1,0,0)$ because matrix

$$
\left(\begin{array}{llll}
0 & 0 & 1 & 1 \\
1 & 1 & 0 & 0
\end{array}\right)
$$

satisfies IOC (Remark 2).

Example 2. $(0,1,1,0) \nsucceq(1,0,0,0)$ and $(1,0,0,0) \nsucceq(0,1,1,0)$ because

$$
\text { neither }\left(\begin{array}{llll}
0 & 1 & 1 & 0 \\
1 & 0 & 0 & 0
\end{array}\right) \text {, nor }\left(\begin{array}{llll}
1 & 0 & 0 & 0 \\
0 & 1 & 1 & 0
\end{array}\right)
$$

satisfies IOC (Remark 3).

From now on, we shall indistinctly denote any $n$-tuple $u \in\{0,1\}^{n}$ by its binary representation $\left(u_{1}, \ldots, u_{n}\right)$ or by its decimal representation $u_{(10}$. Also, we shall denote by " $\leq_{\text {lex }}$ " the usual lexicographic order (truth-table order) between the binary $n$-tuples, with the convention that $0<1$, i.e.,

$$
\left(u_{1}, \ldots, u_{n}\right) \equiv u_{(10}:=\sum_{i=1}^{n} 2^{n-i} u_{i}, u \leq_{l e x} v \Leftrightarrow u_{(10} \leq v_{(10} .
$$


The next corollary (see [2] for the proof) states the relation between the (partial) intrinsic order " $\preceq$ " and the (total) lexicographic order " $\leq_{l e x}$ ".

Corollary 1. For all $u, v \in\{0,1\}^{n}$

$$
u \succeq v \Rightarrow u \leq_{l e x} v, i . e ., \quad u \succeq v \Rightarrow u_{(10} \leq v_{(10 .}
$$

\section{Covering Relation in $\boldsymbol{P}_{n}$}

As is well-known [5], every finite poset is completely determined by its cover relations. Specifically, with respect to our poset $P_{n}$, we say that $v$ is covered by $u$ (or $u$ covers $v$ ), denoted by $v \triangleleft u$ (or $u \triangleright v$ ) if $v \prec u$ and there is no $w \in\{0,1\}^{n}$ such that $v \prec w \prec u$. For instance, in $P_{2}=\left(\{0,1\}^{2}, \preceq\right)$, we have $(1,1) \triangleleft(1,0) \triangleleft(0,1) \triangleleft(0,0)$ because $(1,1) \prec(1,0) \prec(0,1) \prec(0,0)$ (Definition 1 . Theorem 1) with no other elements between them. However, $(1,1) \prec(0,1)$ but $(1,1) \Varangle(0,1)$, because $(1,0)$ is between them. In this section, we provide a simple matrix characterization of the covering relation associated to the intrinsic order. This condition for " $\triangleleft$ ", required for drawing the intrinsic order graph in the next section, will be obviously a particular case of (more restrictive than) the IOC condition for " $\preceq$ ". First, we need the following lemma.

Lemma 1. Let $u, v \in\{0,1\}^{n}$ and $1 \leq i \leq n-1$.

(i) If $v \preceq u$ then $\left(v_{1}, \ldots, v_{i}\right) \preceq\left(u_{1}, \ldots, u_{i}\right)$.

(ii) If $v \preceq u$ and $\left(\begin{array}{lll}u_{1} & \ldots & u_{i} \\ v_{1} & \ldots & v_{i}\end{array}\right)$ has the same number of $\left(\begin{array}{l}1 \\ 0\end{array}\right)$ columns as $\left(\begin{array}{l}0 \\ 1\end{array}\right)$ columns then $\left(v_{i+1}, \ldots, v_{n}\right) \preceq\left(u_{i+1}, \ldots, u_{n}\right)$.

(iii) If $v \preceq u$ and the columns $\left(\begin{array}{l}u_{i} \\ v_{i}\end{array}\right)$ and $\left(\begin{array}{l}u_{i+1} \\ v_{i+1}\end{array}\right)$ are both different from $\left(\begin{array}{l}1 \\ 0\end{array}\right)$, then the matrix obtained by permuting these two columns of $M_{v}^{u}$, keeping its other columns, satisfies IOC.

(iv) If $v \preceq u,\left(\begin{array}{l}u_{i} \\ v_{i}\end{array}\right)=\left(\begin{array}{l}0 \\ 1\end{array}\right),\left(\begin{array}{l}u_{j} \\ v_{j}\end{array}\right)=\left(\begin{array}{l}1 \\ 0\end{array}\right)$ for some $j>i$, and there is no $k$ such that $i<k<j$ and $\left(\begin{array}{l}u_{k} \\ v_{k}\end{array}\right)=\left(\begin{array}{l}1 \\ 0\end{array}\right)$, then the matrix obtained by replacing the $i$-th and $j$-th columns of $M_{v}^{u}$ by $\left(\begin{array}{l}0 \\ 0\end{array}\right)$ or $\left(\begin{array}{l}1 \\ 1\end{array}\right)$, keeping its other columns, satisfies IOC.

Proof. Using the matrix description IOC of the intrinsic order (Theorem 1), the proof is straightforward.

Theorem 2 (Covering relation in $P_{n}$ ). Let $n \geq 1$ and $u, v \in\{0,1\}^{n}$. Then $v \triangleleft u$ if and only if either

$$
\begin{aligned}
& M_{v}^{u}=\left(\begin{array}{llll}
u_{1} & \ldots & u_{n-1} & 0 \\
u_{1} & \ldots & u_{n-1} & 1
\end{array}\right), \text { or } \\
& M_{v}^{u}=\left(\begin{array}{cccccccc}
u_{1} & \ldots & u_{i-1} & 0 & 1 & u_{i+2} & \ldots & u_{n} \\
u_{1} & \ldots & u_{i-1} & 1 & 0 & u_{i+2} & \ldots & u_{n}
\end{array}\right)(1 \leq i \leq n-1),
\end{aligned}
$$

where we assume that in case (3) the $n-1$ first columns of matrix $M_{v}^{u}$ are deleted if $n=1$; while in case (4) the $i-1$ first (the $n-i-1$ last, resp.) columns of matrix $M_{v}^{u}$ are deleted if $i=1$ (if $i=n-1$, resp.). 
Proof. Sufficient condition. First, note that, for both cases (3) and (4), $v \prec u$ since the two corresponding matrices $M_{v}^{u}$ obviously satisfy IOC. So, for both cases, we only need to prove that if $v \preceq w \preceq u$ then $w=u$ or $w=v$.

If (3) holds then using Corollary 1] we have $v \preceq w \preceq u \Rightarrow u \leq_{\text {lex }} w \leq_{\text {lex }} v$ and then $w=u$ or $w=v$.

If (4) holds then using Corollary 11 we have $v \preceq w \preceq u \Rightarrow u \leq_{\text {lex }} w \leq_{\text {lex }} v$ and then two subcases are possible:

$$
\left(w_{1}, \ldots, w_{i+1}\right)=\left\{\begin{array}{l}
\left(u_{1}, \ldots, u_{i-1}, 0,1\right) \\
\left(u_{1}, \ldots, u_{i-1}, 1,0\right)
\end{array}\right.
$$

- If $i=n-1$, then (44)-a is equivalent to say $w=u$ while (4)-b is equivalent to say $w=v$. Then, $w=u$ or $w=v$.

- Otherwise, if $1 \leq i<n-1$, then using Lemma 1 1 ( $i i)$, we have: On one hand, since $v \preceq w$ and matrix $\left(\begin{array}{lllll}w_{1} & \ldots & w_{i-1} & w_{i} & w_{i+1} \\ u_{1} & \ldots & u_{i-1} & 1 & 1\end{array}\right)$ has the same number of $\left(\begin{array}{l}1 \\ 0\end{array}\right)$ columns as $\left(\begin{array}{l}0 \\ 1\end{array}\right)$ columns (namely, 1 if (44)-a holds and 0 if (4)-b holds) then we get $\left(u_{i+2}, \ldots, u_{n}\right) \preceq\left(w_{i+2}, \ldots, w_{n}\right)$. On the other hand, since $w \preceq$ $u$ and matrix $\left(\begin{array}{ccccc}u_{1} & \ldots & u_{i-1} & 0 & 1 \\ w_{1} & \ldots & w_{i-1} & w_{i} & w_{i+1}\end{array}\right)$ has the same number of $\left(\begin{array}{l}1 \\ 0\end{array}\right)$ columns as $\left(\begin{array}{l}0 \\ 1\end{array}\right)$ columns (namely, 0 if (4)-a holds and 1 if (4)-b holds) then we get $\left(w_{i+2}, \ldots, w_{n}\right) \preceq\left(u_{i+2}, \ldots, u_{n}\right)$. So, due to the antisymmetry of the intrinsic order we obtain $\left(w_{i+2}, \ldots, w_{n}\right)=\left(u_{i+2}, \ldots, u_{n}\right)$, and then $w=u$ (if (4)-a holds) or $w=v$ (if (4)-b holds).

Necessary condition. We provide a constructive proof by finding $w \in\{0,1\}^{n}$ yielding $v \supsetneqq w \supsetneqq u$, for all $v \prec u$ such that the pattern of matrix $M_{v}^{u}$ is different from (3) and (4). First, note that since $v \prec u$ we assure (Corollary 1) that $u<_{\text {lex }} v$, i.e., the first column of $M_{v}^{u}$ different from $\left(\begin{array}{l}0 \\ 0\end{array}\right)$ and $\left(\begin{array}{l}1 \\ 1\end{array}\right)$ is $\left(\begin{array}{l}0 \\ 1\end{array}\right)$. Let $\left(\begin{array}{l}u_{i} \\ v_{i}\end{array}\right)$ be this column. Then

$$
M_{v}^{u}=\left(\begin{array}{cccccccc}
u_{1} & \ldots & u_{i-1} & 0 & u_{i+1} & u_{i+2} & \ldots & u_{n} \\
u_{1} & \ldots & u_{i-1} & 1 & v_{i+1} & v_{i+2} & \ldots & v_{n}
\end{array}\right),
$$

where we can assure that $1 \leq i<n$, because if $i=n$ then $M_{v}^{u}$ in (5) would be like (3), and we assume that the $i-1$ first (the $n-i-1$ last, resp.) columns of matrix (5) are deleted if $i=1$ (if $i=n-1$, resp.). The same assumption is established for the $i-1$ first (the $n-i-1$ last, resp.) components of the chosen vectors $w$. We distinguish the following four cases.

(1) $\left(\begin{array}{l}u_{i+1} \\ v_{i+1}\end{array}\right)=\left(\begin{array}{l}0 \\ 0\end{array}\right)$. In this case we choose

$$
\begin{gathered}
w=\left(u_{1}, \ldots, u_{i-1}, 0,1, v_{i+2}, \ldots, v_{n}\right) \text {, so that } v \neq w \neq u \text { and } \\
M_{v}^{w}=\left(\begin{array}{lllllllll}
u_{1} & \ldots & u_{i-1} & 0 & 1 & v_{i+2} & \ldots & v_{n} \\
u_{1} & \ldots & u_{i-1} & 1 & 0 & v_{i+2} & \ldots & v_{n}
\end{array}\right) \text { and } M_{w}^{u}=\left(\begin{array}{llllllll}
u_{1} & \ldots & u_{i-1} & 0 & 0 & u_{i+2} & \ldots & u_{n} \\
u_{1} & \ldots & u_{i-1} & 0 & 1 & v_{i+2} & \ldots & v_{n}
\end{array}\right)
\end{gathered}
$$

satisfy IOC: the first one obviously; the second one due to Lemma 1子( iii).

(2) $\left(\begin{array}{l}u_{i+1} \\ v_{i+1}\end{array}\right)=\left(\begin{array}{l}1 \\ 1\end{array}\right)$. In this case we choose

$$
w=\left(u_{1}, \ldots, u_{i-1}, 1,0, u_{i+2}, \ldots, u_{n}\right) \text {, so that } v \neq w \neq u \text { and }
$$




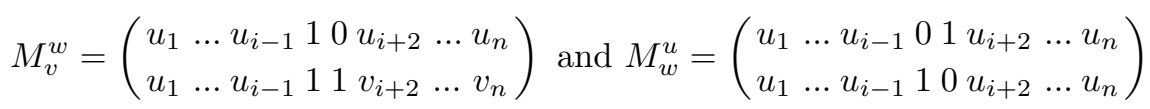

satisfy IOC: the first one due to Lemma 1 $($ iii $)$; the second one obviously.

(3) $\left(\begin{array}{l}u_{i+1} \\ v_{i+1}\end{array}\right)=\left(\begin{array}{l}0 \\ 1\end{array}\right)$. Here we distinguish two subcases:

- (3.1) $M_{v}^{u}$ has no $\left(\begin{array}{l}1 \\ 0\end{array}\right)$ columns. In this subcase we choose

$$
\begin{aligned}
& w=\left(u_{1}, \ldots, u_{i-1}, 0,1, v_{i+2}, \ldots, v_{n}\right) \text {, so that } v \neq w \neq u \text { and }
\end{aligned}
$$

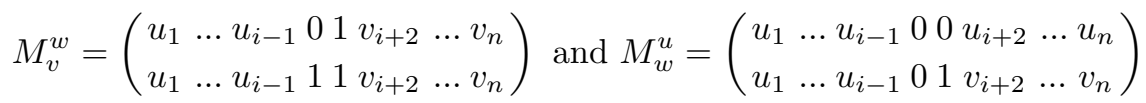

satisfy IOC, since they have no $\left(\begin{array}{l}1 \\ 0\end{array}\right)$ columns.

- (3.2) $M_{v}^{u}$ has at least one $\left(\begin{array}{l}1 \\ 0\end{array}\right)$ column. Let $\left(\begin{array}{l}u_{j} \\ v_{j}\end{array}\right), i+2 \leq j \leq n$, be the left-most $\left(\begin{array}{l}1 \\ 0\end{array}\right)$ column of $M_{v}^{u}$. In this subcase we choose

$$
w=\left(u_{1}, \ldots, u_{i-1}, 0,1, v_{i+2}, \ldots, v_{j-1}, 1, v_{j+1}, \ldots, v_{n}\right), \text { so that } v \neq w \neq u
$$

and with the assumption that the substring $\left(v_{i+2}, \ldots, v_{j-1}\right)$ of $w$ is deleted if $j=i+2$. Then

$$
\begin{gathered}
M_{v}^{w}=\left(\begin{array}{llllllllllll}
u_{1} & \ldots & u_{i-1} & 0 & 1 & v_{i+2} & \ldots & v_{j-1} & 1 & v_{j+1} & \ldots & v_{n} \\
u_{1} & \ldots & u_{i-1} & 1 & 1 & v_{i+2} & \ldots & v_{j-1} & 0 & v_{j+1} & \ldots & v_{n}
\end{array}\right), \\
M_{w}^{u}=\left(\begin{array}{lllllllllllll}
u_{1} & \ldots & u_{i-1} & 0 & 0 & u_{i+2} & \ldots & u_{j-1} & 1 & u_{j+1} & \ldots & u_{n} \\
u_{1} & \ldots & u_{i-1} & 0 & 1 & v_{i+2} & \ldots & v_{j-1} & 1 & v_{j+1} & \ldots & v_{n}
\end{array}\right)
\end{gathered}
$$

satisfy IOC: the first one obviously; the second one due to Lemma 1 $(i v)$.

(4) $\left(\begin{array}{l}u_{i+1} \\ v_{i+1}\end{array}\right)=\left(\begin{array}{l}1 \\ 0\end{array}\right)$. Recall that for all possible cases (1)-(4) we assured that $1 \leq i<n$. Moreover, in this fourth case we can assure that $1 \leq i<n-1$ and $\left(v_{i+2}, \ldots, v_{n}\right) \neq\left(u_{i+2}, \ldots, u_{n}\right)$, because if $i=n-1$ or if $i<n-1$ with $\left(v_{i+2}, \ldots, v_{n}\right)=\left(u_{i+2}, \ldots, u_{n}\right)$ then, in both cases, $M_{v}^{u}$ in (5) would has the pattern (4). In this last case we choose

$$
\begin{gathered}
w=\left(u_{1}, \ldots, u_{i-1}, 0,1, v_{i+2}, \ldots, v_{n}\right) \text {, so that } v \neq w \neq u \text { and } \\
M_{v}^{w}=\left(\begin{array}{lllllllll}
u_{1} & \ldots & u_{i-1} & 0 & 1 & v_{i+2} & \ldots & v_{n} \\
u_{1} & \ldots & u_{i-1} & 1 & 0 & v_{i+2} & \ldots & v_{n}
\end{array}\right) \text { and } M_{w}^{u}=\left(\begin{array}{llllllll}
u_{1} & \ldots & u_{i-1} & 0 & 1 & u_{i+2} & \ldots & u_{n} \\
u_{1} & \ldots & u_{i-1} & 0 & 1 & v_{i+2} & \ldots & v_{n}
\end{array}\right)
\end{gathered}
$$

satisfy IOC: the first one obviously; the second one due to Lemma 1 f $(i v)$.

\section{The Intrinsic Order Graph}

The classical picture of a poset $P$ is its Hasse diagram. This is a directed graph (digraph, for short) whose vertices are the elements of $P$, whose edges are the cover relations, and with the usual convention that if $v \prec u$ then $u$ is drawn 
"above" $v[5]$. For instance, the digraph of $P_{1}$ is $\left.\right|_{1} ^{0}$, since $1 \triangleleft 0$. The next theorem provides us with a fast algorithm for iteratively building up the Hasse diagram of $P_{n}$, for all $n>1$, from the Hasse diagram of $P_{1}$. The binary $n$-tuples are represented by their decimal numbering.

Theorem 3 (Iterative construction of $P_{n}$ from $P_{1}$ ). For all $n>1$, the digraph of $P_{n}=\left\{0, \ldots, 2^{n}-1\right\}$ can be drawn simply by adding to the digraph of $P_{n-1}=\left\{0, \ldots, 2^{n-1}-1\right\}$ its isomorphic copy $2^{n-1}+P_{n-1}=\left\{2^{n-1}, \ldots, 2^{n}-1\right\}$. This addition must be performed placing the powers of 2 at consecutive levels of the Hasse diagram of $P_{n}$. Finally, the edges $(u \triangleright v)$ connecting one vertex $u$ of $P_{n-1}$ with one vertex $v$ of $2^{n-1}+P_{n-1}$ is given by the set of vertex pairs

$$
\{(u, v) / u \triangleright v\}=\left\{\left(u_{(10}, 2^{n-2}+u_{(10}\right) / 2^{n-2} \leq u_{(10} \leq 2^{n-1}-1\right\} .
$$

Proof. For all $n>1$, consider the following partition of $\{0,1\}^{n}$

$$
\begin{aligned}
\{0,1\}^{n} & =\left(\{0\} \times\{0,1\}^{n-1}\right) \cup\left(\{1\} \times\{0,1\}^{n-1}\right), \text { i.e. }, \\
\left\{0, \ldots, 2^{n}-1\right\} & =\left\{0, \ldots, 2^{n-1}-1\right\} \cup\left\{2^{n-1}, \ldots, 2^{n}-1\right\} .
\end{aligned}
$$

On one hand, note that according to IOC (Theorem 11), the subposets of $P_{n}$, $\left(\{0\} \times\{0,1\}^{n-1}, \preceq\right)$ and $\left(\{1\} \times\{0,1\}^{n-1}, \preceq\right)$, are both isomorphic to $P_{n-1}$ in the following sense. For all $\left(u_{1}, \ldots, u_{n-1}\right),\left(v_{1}, \ldots, v_{n-1}\right) \in\{0,1\}^{n-1}$

$$
\begin{aligned}
\left(u_{1}, \ldots, u_{n-1}\right) & \preceq\left(v_{1}, \ldots, v_{n-1}\right) \Leftrightarrow\left(0, u_{1}, \ldots, u_{n-1}\right) \preceq\left(0, v_{1}, \ldots, v_{n-1}\right) \\
& \Leftrightarrow\left(1, u_{1}, \ldots, u_{n-1}\right) \preceq\left(1, v_{1}, \ldots, v_{n-1}\right) .
\end{aligned}
$$

On the other hand, note that according to Theorem 2, we have for all $n>1$

$$
(0, \ldots, 0,1) \triangleright(0, \ldots, 1,0) \triangleright \cdots \triangleright(1,0, \ldots, 0) \text {, i.e., } 2^{0} \triangleright 2^{1} \triangleright \cdots \triangleright 2^{n-1} .
$$

Equations (6) and (7) prove that $P_{n}$ can be iteratively built up by adding to the digraph of $P_{n-1}$ its isomorphic copy $2^{n-1}+P_{n-1}$, placing the powers of 2 at consecutive levels of the Hasse diagram of $P_{n}$. Finally, we prove the last assertion about the edges connecting the two mentioned subposets of $P_{n}$. Let $u \in\{0\} \times\{0,1\}^{n-1} \equiv P_{n-1}, v \in\{1\} \times\{0,1\}^{n-1} \equiv 2^{n-1}+P_{n-1}$ and put $u=\left(0, u_{1}, \ldots, u_{n-1}\right), v=\left(1, v_{1}, \ldots, v_{n-1}\right)$. Using Theorem 2 and taking into account that the pattern (3) is not possible for matrix $M_{v}^{u}$ because $n \geq 2$, we have

$$
\begin{aligned}
& u \triangleright v \Leftrightarrow M_{v}^{u}=\left(\begin{array}{llll}
0 & u_{1} & \ldots & u_{n-1} \\
1 & v_{1} & \ldots & v_{n-1}
\end{array}\right) \text { has the pattern (4) } \\
& \Leftrightarrow\left(\begin{array}{l}
u_{1} \\
v_{1}
\end{array}\right)=\left(\begin{array}{l}
1 \\
0
\end{array}\right) \text { and, if } n>2 \text { then } v_{i}=u_{i} \text { for all } i=2, \ldots, n-1 \\
& \Leftrightarrow u=\left\{\begin{array}{c}
(0,1) \text { if } n=2 \\
\left(0,1, u_{2}, \ldots, u_{n-1}\right) \text { if } n>2
\end{array}, v=\left\{\begin{array}{c}
(1,0) \text { if } n=2 \\
\left(1,0, u_{2}, \ldots, u_{n-1}\right) \text { if } n>2
\end{array}\right.\right.
\end{aligned}
$$




$$
\Leftrightarrow(0,1,0, \stackrel{n-2}{\cdots}, 0)_{(10} \equiv 2^{n-2} \leq u_{(10} \leq 2^{n-1}-1 \equiv\left(0,1,1, \frac{n-2}{\cdots}, 1\right)_{(10}
$$

and

$$
v_{(10}=u_{(10}-2^{n-2}+2^{n-1}=u_{(10}+2^{n-2}
$$

and this concludes the proof.

Fig. 1 illustrates the iterative construction of the digraph of $P_{n}(n=1,2,3,4)$ from $P_{1}$, denoting all the binary $n$-tuples by their decimal numbering.

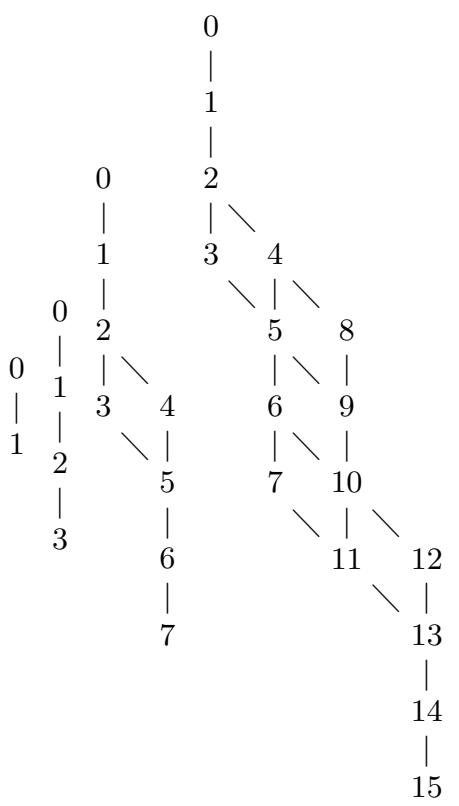

Fig. 1. The intrinsic order graph for $n=1,2,3,4$

\section{References}

1. González, L.: A new method for ordering binary states probabilities in Reliability and Risk Analysis. Lect. Notes Comput. Sc. 2329(1) (2002) 137-146

2. González, L.: N-tuples of 0s and 1s: Necessary and sufficient conditions for intrinsic order. Lect. Notes Comput. Sc. 2667(1) (2003) 937-946

3. González, L., Galván, B., García, D.: Sobre el análisis computacional de funciones Booleanas estocásticas de muchas variables. In: González, L., Sendra, J.R. (eds.): Proc. Primer Encuentro de Álgebra Computacional y Aplicaciones (EACA-95). Santander (1995) 45-55

4. González, L., García, D., Galván, B.J.: An intrinsic order criterion to evaluate large, complex fault trees. IEEE Trans. Reliability 53(3) (2004) 297-305

5. Stanley, R.P.: Enumerative Combinatorics. Volume 1. Cambridge University Press, Cambridge (1997)

6. Stuart, A., Ord, J.K.: Kendall's Advanced Theory of Statistics. Volume 1: Distribution Theory. Oxford University Press, New York (1998) 